Supporting Information

\title{
Scattered light imaging enables real-time monitoring of label-free nanoparticles and fluorescent biomolecules in live cells
}

Fengbang Wang ${ }^{1,2,6}$, Bolei Chen ${ }^{3}$, Bing Yan ${ }^{4}$, Yongguang Yin ${ }^{1,2}$, Ligang $\mathrm{Hu}^{1}$, Yong Liang $^{3,5}$, Maoyong Song ${ }^{1,2,6 *}$, Guibin Jiang ${ }^{1,6}$

${ }^{1}$ Stake Key Laboratory of Environmental Chemistry and Ecotoxicology, Research Center for EcoEnvironmental Sciences, Chinese Academy of Sciences, Beijing 100085, P.R. China.

${ }^{2}$ Key Laboratory of Environmental Nanotechnology and Health Effects, Research Center for EcoEnvironmental Sciences, Chinese Academy of Sciences, Beijing 100085, P.R. China.

${ }^{3}$ Hubei Key Laboratory of Environmental and Health Effects of Persistent Toxic Substances, Jianghan University, Wuhan 430056, P.R. China.

${ }^{4}$ Institute of Environmental Research at Greater Bay, Key Laboratory for Water Quality and Conservation of the Pearl River Delta, Ministry of Education, Guangzhou University, Guangzhou 510006, P.R. China.

${ }^{5}$ Institute of Environment and Health, Jianghan University, Wuhan 430056, P.R. China.

${ }^{6}$ University of Chinese Academy of Sciences, Beijing 100049, P.R. China 


\section{Experimental Section}

Chemicals. All AgNPs used in this study were purchased from nanoComposix (San Diego, USA). Multiwalled carbon nanotubes and silver nanowires were purchased from Xianfeng Nanocompany (Nanjing, China). Lanthanum(III) oxide nanoparticles (La2O3NPs), pyrrolidine tris-acid modified fullerene nanoparticles, zirconium(IV) oxide nanoparticles (ZrO2NPs), zinc oxide nanoparticles (Zn2O3NPs), ferrosoferric oxide nanoparticles (Fe3O4NPs), silica nanoparticles (SiO2NPs), copper(II) oxide nanoparticles (CuONPs), titanium dioxide nanoparticles (TiO2NPs), and golden nanoparticles (AuNPs) were obtained from Sigma-Aldrich company (Shanghai, China). Mouse macrophages (J774A.1) were purchased from China Infrastructure of Cell Line Resource (Beijing). Dulbecco's modified Eagle's medium (DMEM), penicillin-streptomycin solution, and fetal bovine serum (FBS) were purchased from Gibco (Carlsbad, USA). Fluorescent dyes, including Hoechst 33342, SYTO 9, SYTO 82, and Lysotracker Red probe were obtained from Invitrogen (Carlsbad, USA). All aqueous solutions were prepared using high-purity deionized water.

Preparation and characterization of NPs. A total of $10 \mathrm{mg}$ of each nanomaterial was suspended in $20 \mathrm{~mL}$ of deionized water and sonicated for $20 \mathrm{~min}$ at $4{ }^{\circ} \mathrm{C}$. The concentration of NPs in supernatant was measured by gravimetric method. The suspension of each NPs was diluted to 0.01 $\mathrm{mg} / \mathrm{mL}$, then $5 \mu \mathrm{L}$ of the diluent was deposited on an ultrafine carbon support film on a copper grid and dried overnight in a dust-free box. The morphology and structure of the NPs were imaged using transmission electron microscopy (TEM) (JEM-2100F, Toyota, Japan).

Cell culture and NPs exposure. J774A.1 macrophages were cultured in DMEM supplemented with $10 \%$ FBS (heat inactivated), and $100 \mathrm{U} / \mathrm{mL}$ penicillin and streptomycin. Cells were cultured in $100 \mathrm{~mm}$ tissue culture plates at $37{ }^{\circ} \mathrm{C}$ in a $5 \% \mathrm{CO}_{2}$ humidified atmosphere until $80-90 \%$ cell confluence. Exposure of NPs was performed by adding fresh culture medium containing NPs into the cell culture plates. The dose of NPs was adjusted by diluting the NPs stock solution with cell culture medium.

Confocal imaging of living cells. Cells were treated with fluorescent dyes for 20 min at $37^{\circ} \mathrm{C}$ with $5 \% \mathrm{CO}_{2}$. After washing three times with $1 \times$ phosphate-buffer saline (PBS), the cells were imaged using a Leica SP5 confocal laser scanning microscopy (German).

Scattering light imaging (SLi) of NPs in live cells. Leica SP5 confocal laser scanning microscopy was used for SLi of NPs in live cells. SLi was performed in the fluorescence mode on the confocal microscope. Imaging conditions were $405 \mathrm{~nm}$ excitation and 400-410 nm detection, $488 \mathrm{~nm}$ excitation and 483-493 nm detection, and $543 \mathrm{~nm}$ excitation and 538-548 $\mathrm{nm}$ detection.

Measurement of single AgNP in J774A.1 macrophages using SLi. To image single AgNP, AgNPs were fixed in polyacrylamide gel. Briefly, AgNPs $(5 \mu \mathrm{g} / \mathrm{mL})$ were added into pre-warmed Glycerol Jelly Mounting Medium $\left(45^{\circ} \mathrm{C}\right)$. The AgNPs suspension was subsequently vortexed and sonicated at $45{ }^{\circ} \mathrm{C}$ for $5 \mathrm{~min}$. Then $200 \mu \mathrm{L}$ of the suspension was transferred into the center of a glass bottom culture dish and cooled at room temperature to form a gel. The gel contained AgNPs is then imaged using SLi. The individual AgNPs are clearly distinguishable in the gel and appeared as a sharp-edged green dot (Figure S5). The size and shape of these single particles in SLi images of gel are used as criterion to determine single AgNP internalized inside J774A.1 macrophages. To 
measure the size of single AgNP in J774A.1 macrophages using SLi, AgNPs are exposed to J774A.1 macrophages at different concentrations and imaged at different times. Only dots with complete external shape in the SLi images were selected for physical cross section size measurements. The average diameter of each size of AgNPs is determined by counting 100 single particles in SLi images at random.

Simultaneous SLi and florescence imaging. The scattering light signal from AgNPs and fluorescence from fluorescent-labeled biomolecules were recorded in two independent fluorescence channels simultaneously in the fluorescence mode of confocal microscopy, in which PMT1, PMT2 and PMTtrans were opened simultaneously. PMT1 was used to detect scattered light, PMT2 was used to detect fluorescence, and PMTtrans was used to observe the cell morphology. 

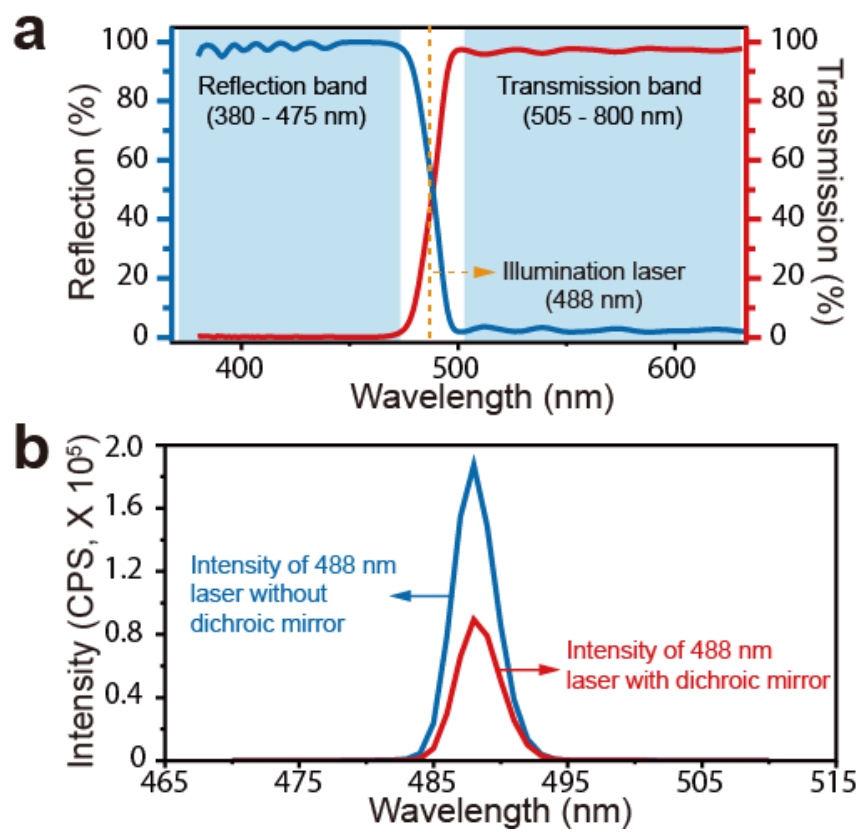

Figure S1. a) Wavelength of the $488 \mathrm{~nm}$ laser is between the reflection band (380 - $475 \mathrm{~nm})$ and transmission band (505- $800 \mathrm{~nm}$ ) of the DM with a cut-on wavelength of $490 \mathrm{~nm}$ (DM490). b) Intensity spectra of the $488 \mathrm{~nm}$ illumination laser with and without passing through MD490. 

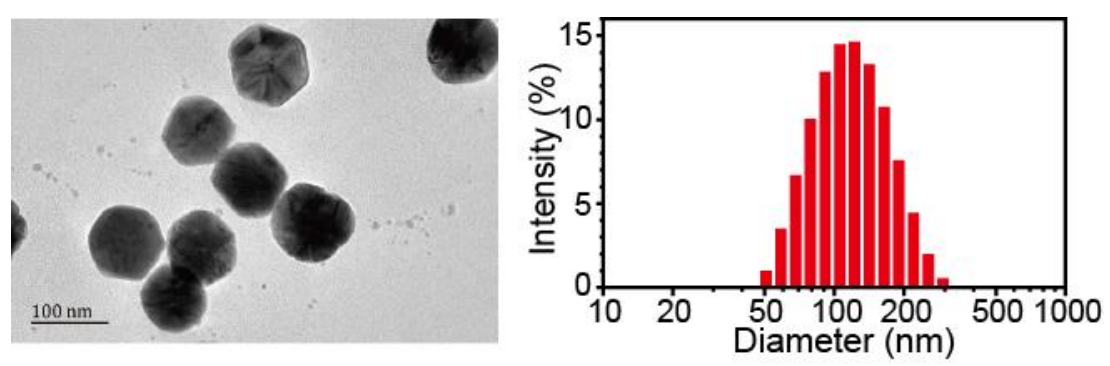

Figure S2. TEM image of $100 \mathrm{~nm}$ citric acid-coated AgNPs (CA-AgNPs) (left) and its size distribution (right). The size distribution of CA-AgNPs is determined by counting 100 single particles in TEM images at random. 

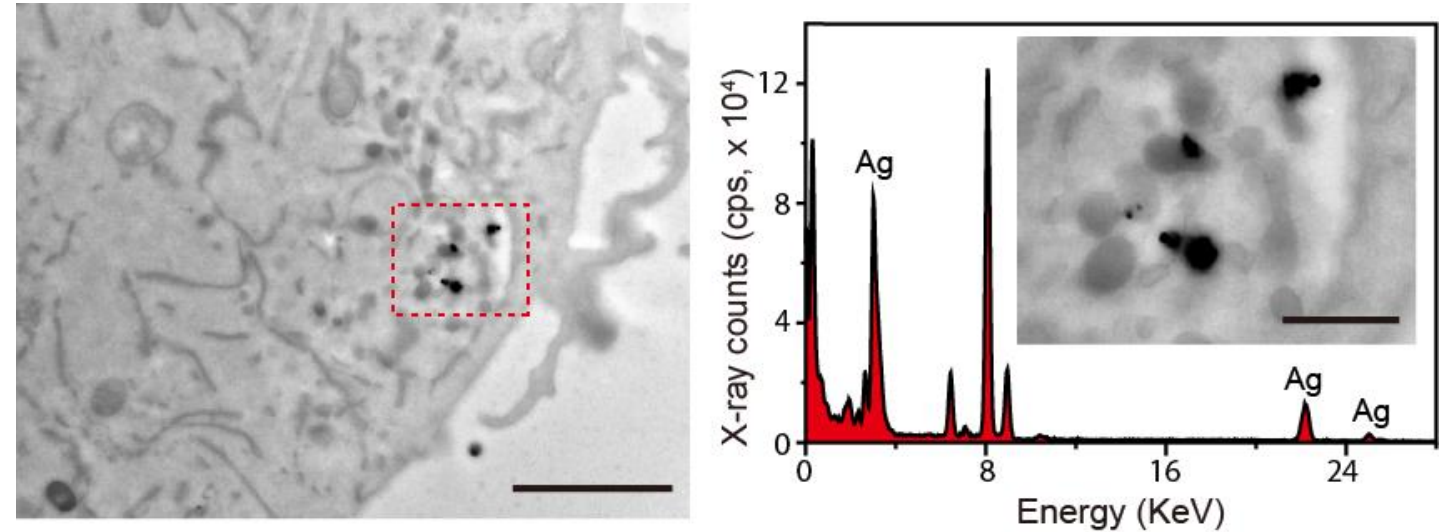

Figure S3. TEM image (left) and X-ray analysis (right) showing the internalization of CA-AgNPs $(100 \mathrm{~nm})$ in J774A.1 macrophage. 

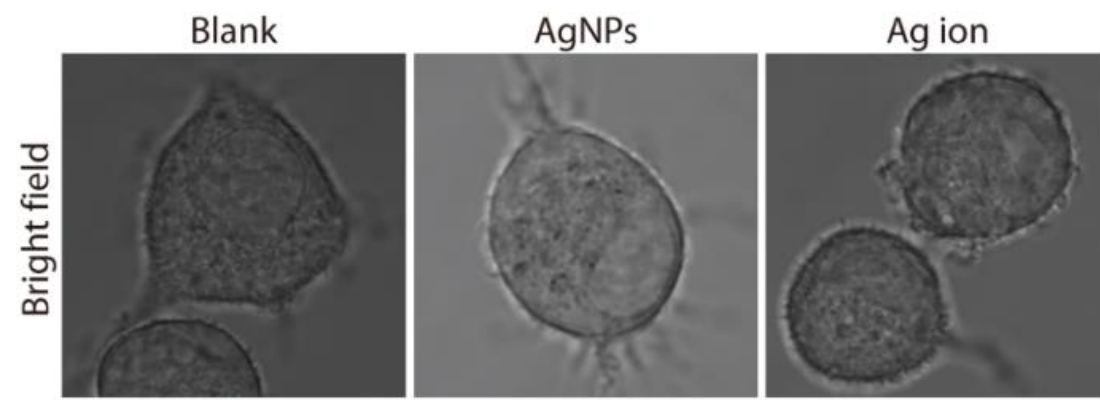

SYTO 9 Green
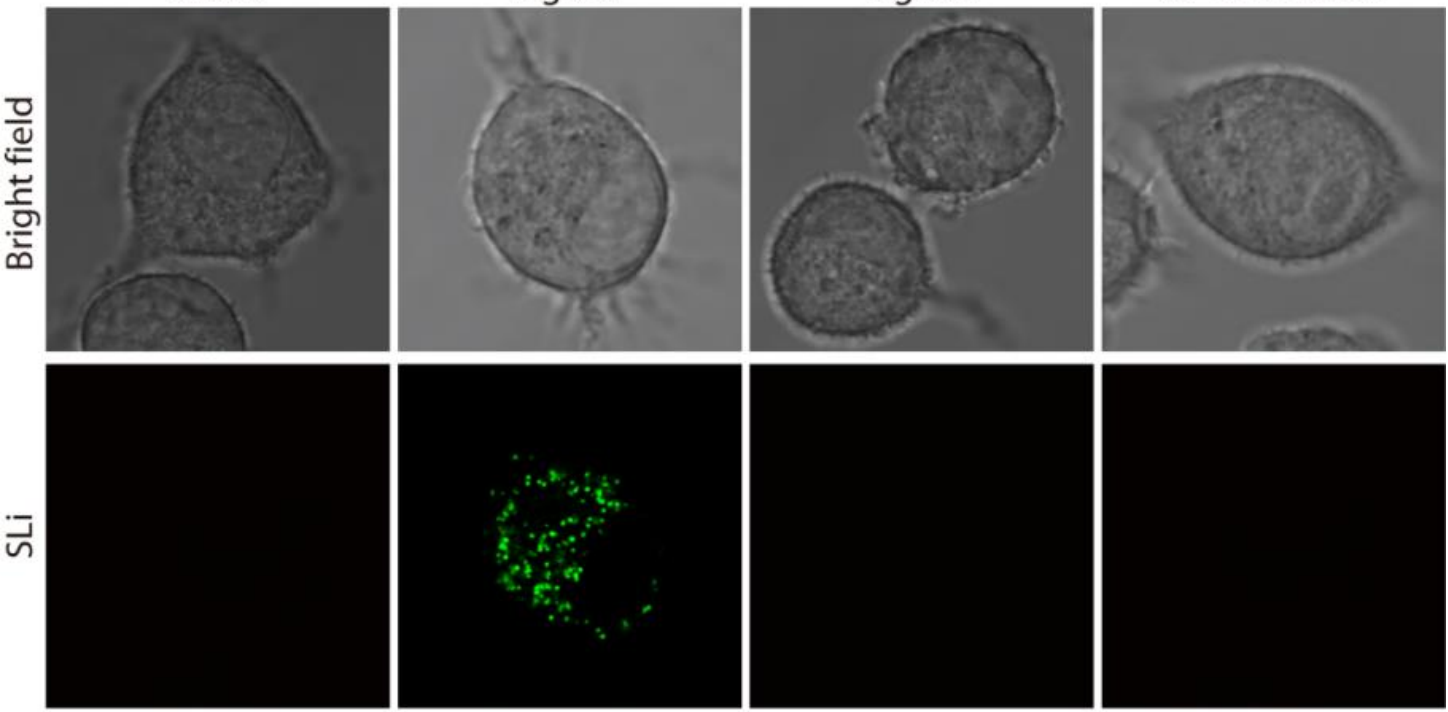

Excitation wavelength $=488 \mathrm{~nm}$

Emission wavelength $=483-493 \mathrm{~nm}$

Figure S4. Bright field images (top row) and SLi images (bottom row) of J774A.1 macrophage treated with CA-AgNPs, silver ions, SYTO 9 Green. Results showed that silver ions, SYTO 9 Green, and subcellular organelle had no effects on the scattered light imaging of CA-AgNPs. 


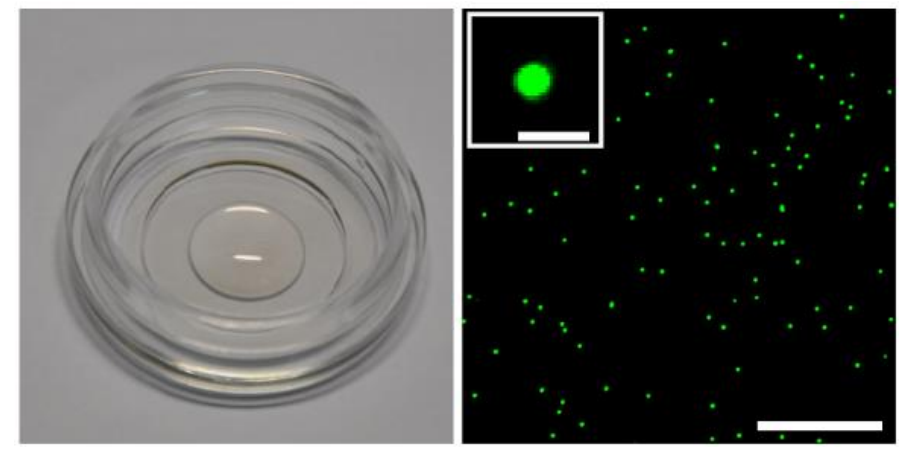

Figure S5. SLi images for CA-AgNPs $(100 \mathrm{~nm})$ which were fixed in polyacrylamide gel. The inset is representative SLi images of a single AgNP. Scale bars are $20 \mu \mathrm{m}$ in SLi image and $1 \mu \mathrm{m}$ in inset, respectively. 


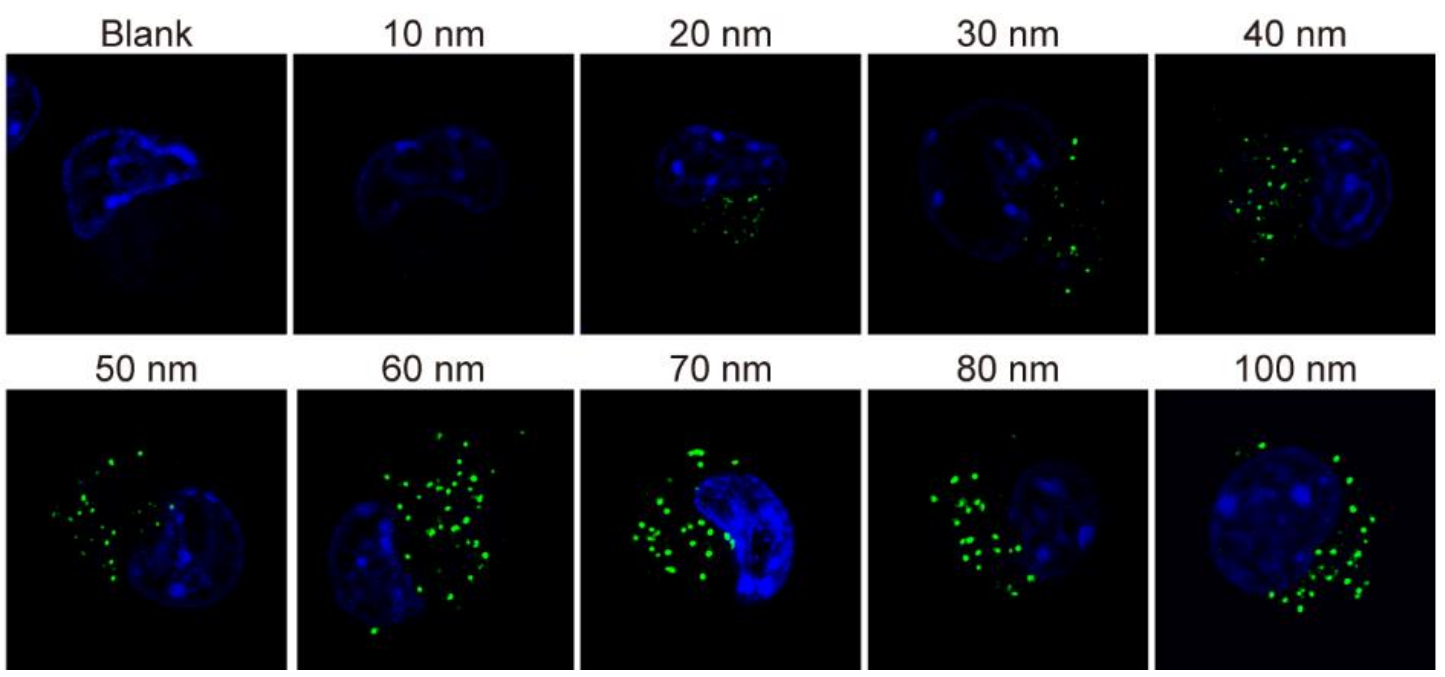

Figure S6. Merged images of SLi image of CA-AgNPs (green) with different size and fluorescence image of Hoechst 33342 dyed cell nucleus (blue). SLi and fluorescence imaging are performed simultaneously in two independent fluorescence channels. SLi is performed under $488 \mathrm{~nm}$ laser excitation with emission set to $483-493 \mathrm{~nm}$, and fluorescence imaging is performed under $405 \mathrm{~nm}$ laser excitation with emission set to $425-500 \mathrm{~nm}$. Scale bar is $10 \mu \mathrm{m}$. 

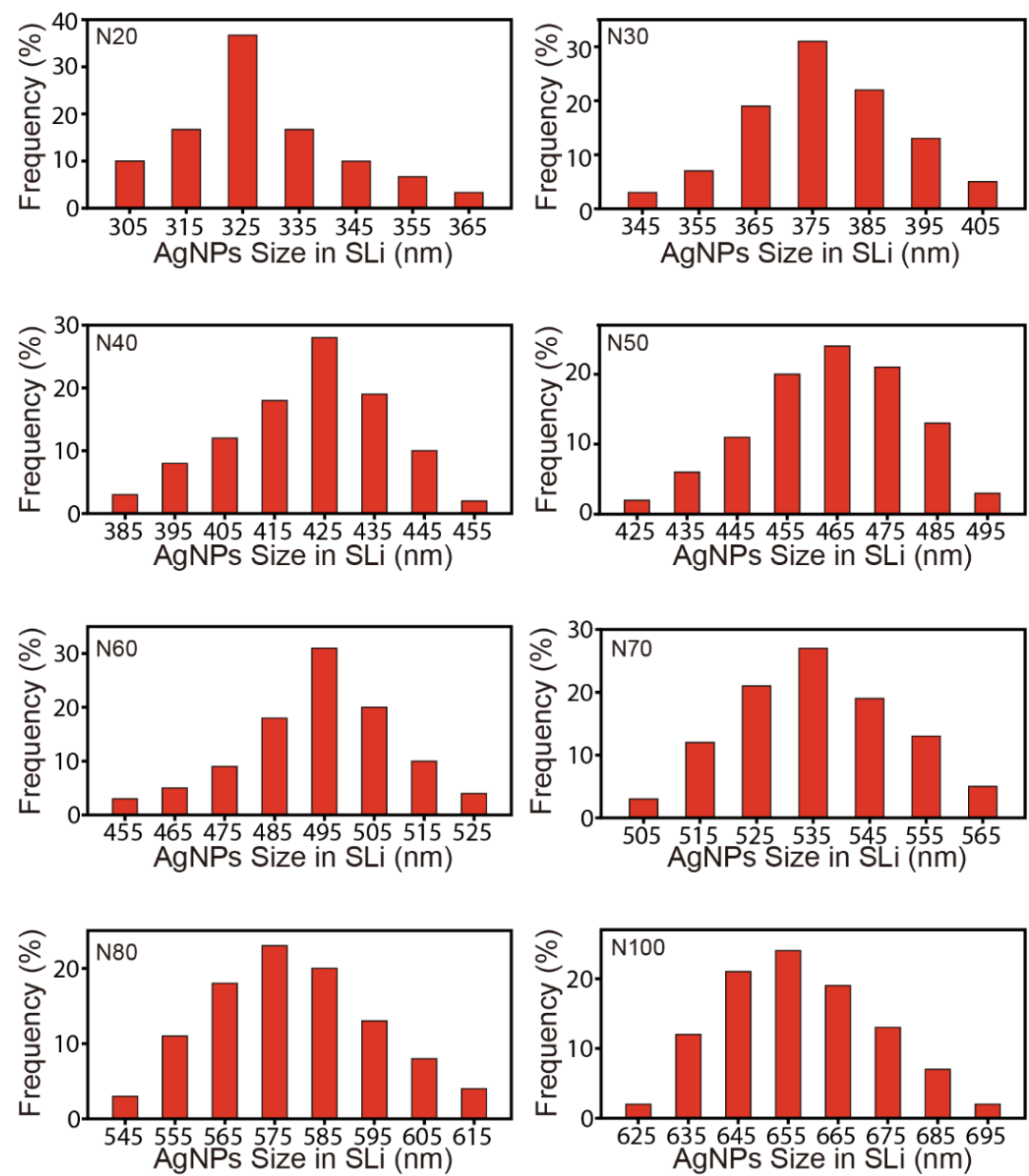

Figure S7. The size distribution of CA-AgNPs in J774A.1 macrophage. The size values were obtained by counting 100 single particle at random in SLi images of J774A.1 macrophage treated with CA-AgNPs. 

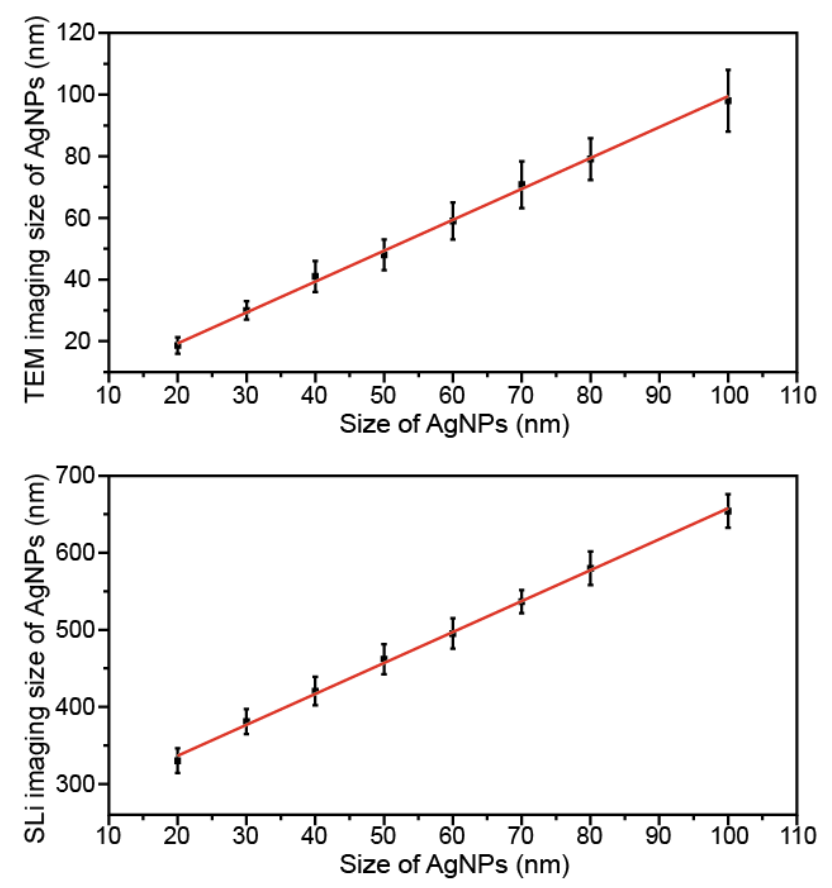

Figure S8. Mean value of the size of each CA-AgNP measured in TEM images (top) and in SLi images (bottom) vs its commercial marked size. The size values were obtained by counting 100 single particle in TEM and SLi images at random. Data are averages \pm SD $(n=3)$. 

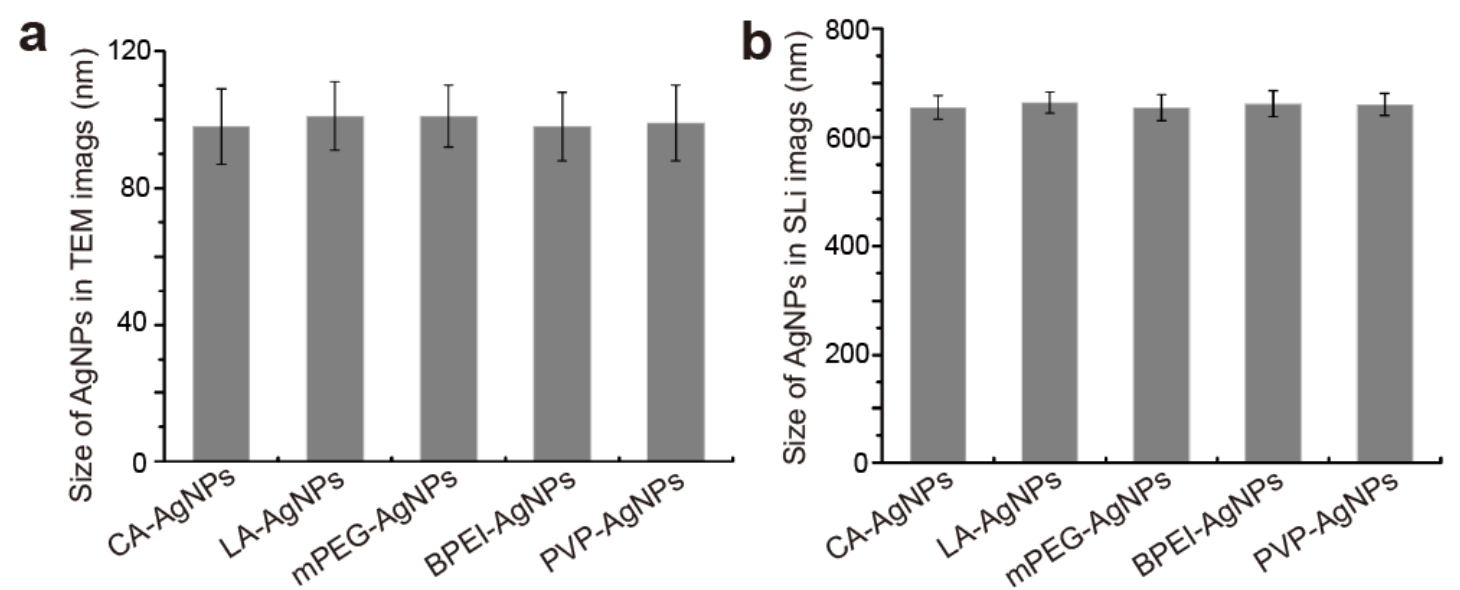

Figure S9. Mean value of sizes of different AgNPs in TEM (a) and SLi (b) images. The average diameter of AgNPs types is determined by counting 100 single particles in SLi and TEM images at random. Data are averages $\pm \mathrm{SD}(\mathrm{n}=3)$. 


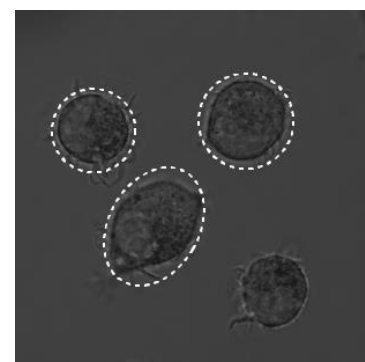

Bright field image

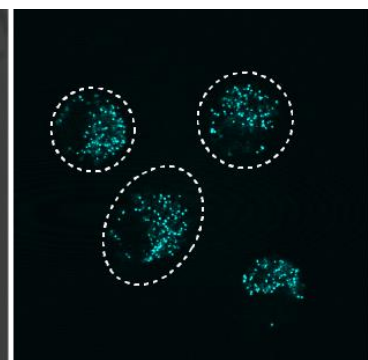
Excitation wavelength $=405 \mathrm{~nm}$

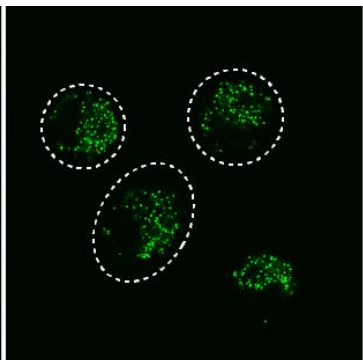
Excitation wavelength $=488 \mathrm{~nm}$

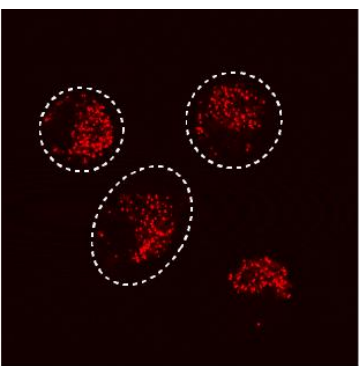

Excitation wavelength $=543 \mathrm{~nm}$ Exctation wavelength $=543 \mathrm{~nm}$

Figure S10. The colour of the CA-AgNPs inside macrophage under different the excitation and detection wavelength (Cyan, $405 \mathrm{~nm}$ excitation and $400-410 \mathrm{~nm}$ detection; Green, $488 \mathrm{~nm}$ excitation and 483 - $493 \mathrm{~nm}$ detection; Red, $543 \mathrm{~nm}$ excitation and 538 - $548 \mathrm{~nm}$ detection). 

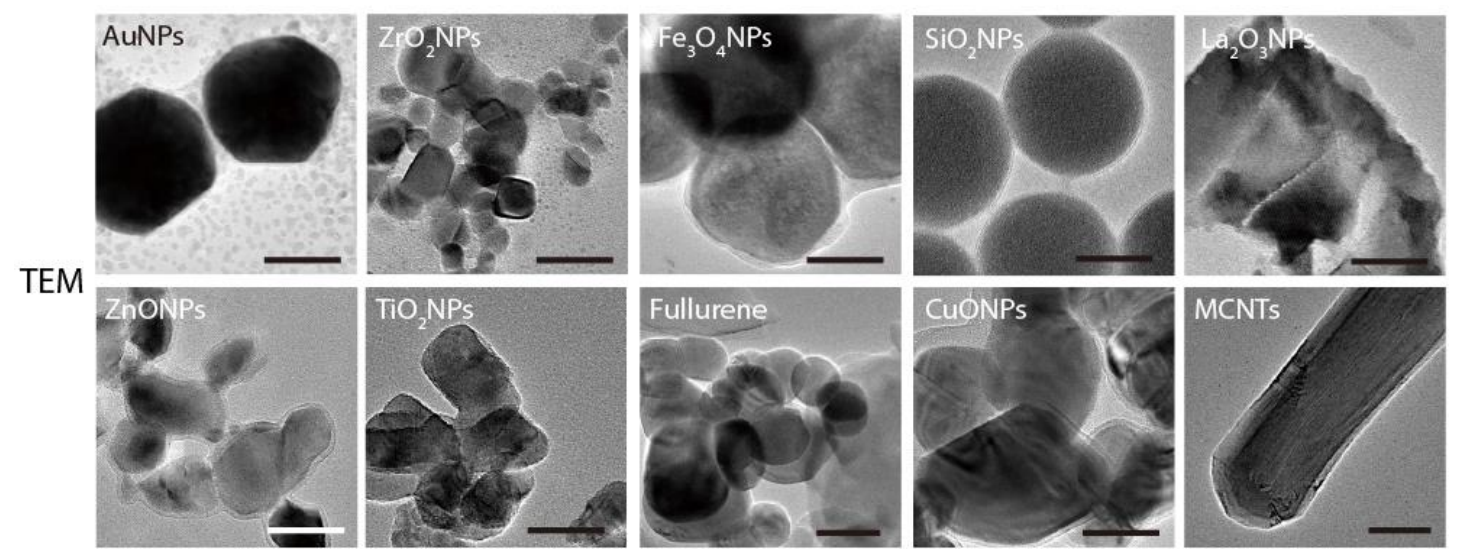

MCNTs

Figure S11. TEM images of 10 types of nanoparticles. Scale bar is $100 \mathrm{~nm}$. 